\title{
Arterial stiffness determined according to the cardio-ankle vascular index is associated with paroxysmal atrial fibrillation: a cross-sectional study
}

\author{
Toru Miyoshi, ${ }^{1}$ Masayuki Doi, ${ }^{2}$ Yoko Noda, ${ }^{3}$ Yuko Ohno, ${ }^{3}$ Kosuke Sakane, ${ }^{4}$ \\ Shigeshi Kamikawa, ${ }^{4}$ Youko Noguchi, ${ }^{4}$ Hiroshi Ito ${ }^{3}$
}

${ }^{1}$ Department of Cardiovascular Therapeutics, Okayama University Graduate School of Medicine, Dentistry and Pharmaceutical Sciences, Okayama, Japan

${ }^{2}$ Department of Cardiology, Kagawa Prefectural Central Hospital, Kagawa, Japan ${ }^{3}$ Department of Cardiovascular Medicine, Okayama University Graduate School of Medicine, Dentistry and Pharmaceutical Sciences, Okayama, Japan ${ }^{4}$ Department of Cardiology, Sumitomo Besshi Hospital, Niihama, Japan

\section{Correspondence to} Dr Toru Miyoshi, Department of Cardiovascular Therapeutics, Okayama University Graduate School of Medicine, Dentistry and Pharmaceutical Sciences, 2-5-1, Shikata-cho, Okayama, Okayama 700-8558, Japan; miyoshit@cc.okayama-u.ac.jp

Received 16 January 2014 Revised 6 March 2014 Accepted 12 April 2014

\section{(1) crossanat}

To cite: Miyoshi T, Doi M, Noda $Y$, et al. Heart Asia 2014;6:59-63.

doi:10.1136/heartasia-2014010503

\section{ABSTRACT}

Background Several lines of evidence suggest that atrial fibrillation (AF) may be a consequence of vascular disease. We investigated the relationship between cardio-ankle vascular index (CAVI), a new index of arterial stiffness, and the presence of paroxysmal $\mathrm{AF}$ (PAF).

Methods and results 181 outpatients (91 patients with PAF and 90 age- and gender-matched subjects without PAF) were analysed for their sinus rhythm. The CAVI was significantly higher in patients with PAF than in subjects without PAF $(9.0 \pm 1.0$ vs $8.7 \pm 0.8, p<0.01)$. In all subjects, the CAVI was significantly correlated with the left ventricular mass index $(r=0.30, p<0.01)$, left atrial diameter $(r=0.22, p<0.01)$, and augmentation index, a parameter of wave reflection $(r=0.32, p<0.01)$, in addition to age, systolic blood pressure and pulse pressure. Logistic analysis demonstrated that the CAVI was independently associated with PAF even after adjustment for confounding factors. The adjusted OR of PAF was 1.8 for each unit increase in the CAVI $(p=0.01)$.

Conclusions Our finding suggests that increased arterial stiffness may be involved in the maintenance of $\mathrm{AF}$.

\section{BACKGROUND}

Atrial fibrillation (AF) is the most common chronic arrhythmia in adults and is associated with an increased risk of mortality from cardiovascular events. ${ }^{12}$ Although several clinical risk factors for AF have been identified, ${ }^{3-6}$ further evaluation of modifiable risk factors is required to prevent the development of AF. A cohort study showed that carotid intima-media thickness is associated with the new onset of $\mathrm{AF}^{7}$ In a case-control study, carotid intima-media thickness as well as arterial stiffness assessed by carotid-femoral pulse wave velocity (PWV) was associated with lone $\mathrm{AF}^{8}$ Increased arterial stiffness, which leads to increased cardiac pressure load, ventricular hypertrophy and left atrial enlargement, could be the perpetuation of $\mathrm{AF}^{9-11}$ Thus, the interaction of vascular function and cardiac load may play a critical role in the maintenance of AF.

The cardio-ankle vascular index (CAVI) has been developed for the quantitative evaluation of vascular wall stiffness in the aorta, femoral arteries and tibial artery by measuring PWV and blood pressure. ${ }^{12}$ Indeed, the CAVI correlates with other cardiovascular risk markers, such as intima-media thickening and coronary atherosclerosis, thus reflecting the degree of atherosclerotic change. ${ }^{13}$ Arterial stiffness affects wave reflection, which results when a forward pressure wave created by ventricular contraction encounters a site of impedance. $^{14} 15$ Thus, increased arterial stiffness can induce left ventricular (LV) hypertrophy and left atrial dilation. ${ }^{16}$

We previously showed that the augmentation index is significantly associated with the presence of paroxysmal $\mathrm{AF}^{17}$ From this, we hypothesised that the CAVI may also be associated with AF. As an examination of the CAVI in patients with chronic AF is technically problematic, we analysed the CAVI in subjects with and without paroxysmal $\mathrm{AF}$ in this study.

\section{METHODS}

\section{Ethics statement}

The experimental procedures and potential risks were explained prior to the study. The Institutional Review Board of Okayama University and Sumitomo Besshi Hospital approved this study and informed consent was obtained from each subject. The study conformed to the principles of the Declaration of Helsinki.

\section{Subjects}

We enrolled 91 outpatients with a history of paroxysmal AF and 90 age- and gender-matched subjects without a history of paroxysmal AF, all of whom visited Sumitomo Besshi Hospital or Okayama University Hospital from October 2009 to May 2011. Paroxysmal AF was diagnosed according to the American College of Cardiology/American Heart Association/European Society of Cardiology OR ACC/AHA/ESC 2006 Guidelines. ${ }^{18}$ Paroxysmal $\mathrm{AF}$ was documented at least once with $24 \mathrm{~h}$ holter monitoring or at clinic 3 months before the enrolment. All subjects were in sinus rhythm at the time of measuring the CAVI and augmentation index. Patients were excluded from the study if they had any of the following conditions: (1) long standing persistent AF; (2) renal insufficiency (serum creatine $>2.0 \mathrm{mg} / \mathrm{mL}$ ); (3) acute coronary syndrome; (4) severe valvular disease and prosthetic valves; (5) decreased LV systolic function (LV ejection fraction $<50 \%)$; or (6) ankle-brachial index $<0.9$.

\section{CAVI measurement}

The CAVI was calculated automatically using a VaSera VS-1000 Vascular Screening System (Fukuda Denshi, Tokyo, Japan) from the blood pressure and PWV, while simultaneously monitoring the 
Table 1 Patient characteristics

\begin{tabular}{|c|c|c|c|}
\hline & $\begin{array}{l}\text { Patients without paroxysmal AF } \\
(n=90)\end{array}$ & $\begin{array}{l}\text { Patients with paroxysmal AF } \\
(n=91)\end{array}$ & p Value \\
\hline Age (years) & $69 \pm 7$ & $70 \pm 8$ & NS \\
\hline Male, n (\%) & $51(57)$ & $55(60)$ & NS \\
\hline Height $(\mathrm{cm})$ & $159 \pm 8$ & $160 \pm 7$ & NS \\
\hline Body mass index $\left(\mathrm{kg} / \mathrm{m}^{2}\right)$ & $23.6 \pm 3.1$ & $23.5 \pm 3.6$ & NS \\
\hline Hypertension, n (\%) & $56(63)$ & $49(53)$ & NS \\
\hline Hyperlipidaemia, n (\%) & $44(49)$ & $27(29)$ & $<0.01$ \\
\hline Diabetes mellitus, $n$ (\%) & $15(17)$ & $16(17)$ & NS \\
\hline \multicolumn{4}{|l|}{ Medications, n (\%) } \\
\hline ACEI/ARB & $35(39)$ & $30(33)$ & NS \\
\hline Calcium channel blockers & $27(30)$ & $35(38)$ & NS \\
\hline$\beta$-Blockers & $5(6)$ & $20(21)$ & 0.001 \\
\hline Statins & $21(22)$ & $19(20)$ & NS \\
\hline Digitalis & $0(0)$ & $15(16)$ & $<0.01$ \\
\hline Antiarrhythmic drugs & $1(1)$ & $51(55)$ & $<0.01$ \\
\hline
\end{tabular}

electrocardiogram and heart sounds. ${ }^{12} 19$ PWV was calculated by dividing the distance from the aortic valve to the ankle artery by the sum of the time between the aortic valve closing sound and the notch of the brachial pulse wave and the time between the rise of the brachial pulse wave and the ankle pulse wave. The CAVI was determined by the following equation: $\mathrm{CAVI}=\mathrm{a}\left[(2 \rho / \Delta \mathrm{P}) \times \ln (\mathrm{Ps} / \mathrm{Pd}) \times \mathrm{PWV}^{2}\right]+\mathrm{b}$, where Ps and Pd are the systolic and diastolic blood pressure, respectively; PWV is between the heart and ankle; $\Delta \mathrm{P}$ is $\mathrm{Ps}-\mathrm{Pd} ; \rho$ is blood density; and $a$ and $b$ are constants. The average of the right and left CAVI values was used for analysis.

\section{Blood pressure measurements and pulse wave analysis}

After a 5-min rest and with the subject seated, brachial blood pressure was measured using an automatic cuff oscillometric device and the average of two readings was used. Next, the radial pulse wave was examined at the wrist using applanation tonometry with a high-fidelity micromanometer (HEM-9000AI, Omron Healthcare Co., Kyoto, Japan), as previously described. ${ }^{16}$

\section{Transthoracic echocardiography}

All echocardiographic data were obtained by experienced sonographers and interpreted by an experienced staff echocardiographer. Comprehensive examinations were performed including $\mathrm{M}$-mode, 2D, conventional Doppler and colour Doppler echocardiography. Measurements were made according to the guidelines of the American Society of Echocardiography. ${ }^{20}$ The left atrial dimensions were assessed by M-mode echocardiography and LV mass was calculated using the Penn convention. ${ }^{21}$ The LV mass index was calculated by dividing the LV mass by the body surface area. The LV ejection fraction was also examined as a measure of cardiac function.

\section{Statistical analysis}

Data are summarised as mean \pm SD for quantitative variables. Categorical variables were compared between groups by $\chi^{2}$ analysis, and continuous variables were compared between two groups by unpaired $\mathrm{t}$ test. Bivariate correlation was assessed using Pearson's correlation analysis. Multiple logistic regression analysis was performed to evaluate the relationships between paroxysmal AF and increasing CAVI, aspects of blood pressure and echocardiographic parameters. The sample size was determined on the basis of the estimated CAVI values reported in our other study. ${ }^{16}$ We assumed a CAVI of $8.8 \pm 1.0($ mean \pm SD) in the control group and $9.0 \pm 1.0$. Using a two-sided test for differences in independent binominal proportions with an $\alpha$ level of 0.05 , we calculated that 172 patients (86 in each group)

Table 2 Haemodynamic and echocardiographic parameters

\begin{tabular}{|c|c|c|c|}
\hline & $\begin{array}{l}\text { Patients without paroxysmal AF } \\
(n=90)\end{array}$ & $\begin{array}{l}\text { Patients with paroxysmal AF } \\
(n=91)\end{array}$ & p Value \\
\hline Systolic blood pressure (mm Hg) & $135 \pm 13$ & $137 \pm 18$ & NS \\
\hline Diastolic blood pressure $(\mathrm{mm} \mathrm{Hg})$ & $73 \pm 10$ & $73 \pm 10$ & NS \\
\hline Mean atrial blood pressure $(\mathrm{mm} \mathrm{Hg})$ & $94 \pm 10$ & $94 \pm 11$ & NS \\
\hline Pulse pressure $(\mathrm{mm} \mathrm{Hg})$ & $63 \pm 11$ & $62 \pm 16$ & NS \\
\hline Heart rate (bpm) & $70 \pm 10$ & $64 \pm 10$ & $<0.01$ \\
\hline Left ventricular mass index $\left(\mathrm{g} / \mathrm{m}^{2}\right)$ & $91.4 \pm 20.1$ & $99.8 \pm 25.5$ & NS \\
\hline Left ventricular ejection fraction (\%) & $70.5 \pm 7.7$ & $71.1 \pm 7.6$ & NS \\
\hline Left atrial diameter (mm) & $35 \pm 5$ & $39 \pm 6$ & $<0.001$ \\
\hline
\end{tabular}

Data are expressed as the mean \pm SD.

$\mathrm{AF}$, atrial fibrillation; NS, not significant. 
Figure 1 Cardio-ankle vascular index (A) and radial augmentation index (B) values in patients with or without paroxysmal atrial fibrillation (AF). Data are presented as mean+SD.
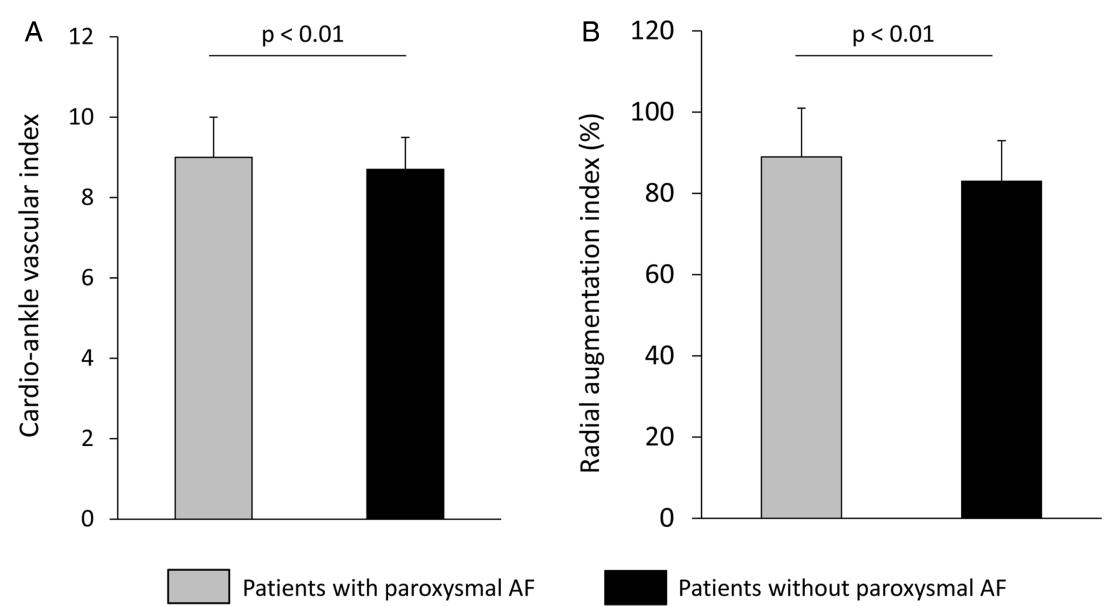

would have $90 \%$ power to detect a difference in the CAVI between two groups. Differences at $\mathrm{p}<0.05$ were considered significant. Data were analysed using SPSS V.17.0 for Windows (SPSS Inc., Chicago, Illinois, USA).

\section{RESULTS}

Table 1 shows the baseline characteristics of the AF and control groups. There were no differences between the groups with regard to age, gender, height, body mass index, prevalence of hypertension and diabetes, whereas the control group included a lower percentage of subjects with hyperlipidaemia than the paroxysmal AF group. Systolic blood pressure, diastolic blood pressure, mean arterial pressure and pulse pressure were comparable between the two groups (table 2). The heart rate in patients with paroxysmal AF was lower than that in patients without paroxysmal AF (table 2). Regarding the drugs administered, the use of $\beta$-blockers, digitalis and antiarrhythmic drugs was higher in patients with paroxysmal AF than in those without paroxysmal AF. There were no significant differences between the two groups in the use of ACE inhibitors and/or angiotensin receptor blockers, calcium channel blockers, and statins. As for echocardiographic parameters, the left atrial diameter was significantly larger in patients with paroxysmal AF.

The CAVI value in patients with paroxysmal AF was significantly higher than that in subjects without paroxysmal AF $(9.0$ \pm 1.0 vs $8.7 \pm 0.8, p<0.01$; figure 1 ). The radial augmentation index value for patients with paroxysmal AF was significantly higher than for subjects without paroxysmal AF $(89 \pm 12 \%$ vs 83 $\pm 10 \%, \mathrm{p}<0.01$; figure 1 ).

Table 3 shows the associations between the CAVI and other parameters of blood pressure, biochemical markers and echocardiography. The CAVI was significantly and positively correlated with age, systolic blood pressure and pulse pressure, and was significantly and negatively correlated with high-density lipoprotein cholesterol. Regarding echocardiographic parameters, the CAVI was significantly and positively correlated with LV mass index and left atrial diameter (figure 2). Furthermore, the CAVI was significantly associated with radial augmentation index (figure 3).

We next carried out a multivariate analysis to evaluate the relative associations of the CAVI, individual blood pressure parameters, LV mass index and left atrial diameter with paroxysmal $\mathrm{AF}$ in a model adjusted for age, gender, heart rate, and use of antihypertensive and antiarrhythmic drugs. In this model, increases in the CAVI and left atrial diameter significantly associated with an increased risk of paroxysmal AF (table 4).
Multiple logistic analysis revealed that the OR of paroxysmal AF was elevated $\sim 1.8$ for each unit increase in the CAVI (table 4 ).

\section{DISCUSSION}

This study showed that an increase in arterial stiffness, as measured by the CAVI, was independently and positively associated with paroxysmal AF. Even after adjusting for other relevant factors, patients with paroxysmal AF had significantly higher CAVI values than did those without paroxysmal AF. These findings suggest a potential role of arterial structural and functional remodelling in the pathogenesis of AF.

Our data are in good agreement with those from a previous study showing increased arterial stiffness assessed by carotidfemoral PWV in patients with lone $\mathrm{AF}^{8}$ Ours is the first study to evaluate the association between the CAVI and AF. Regarding the mechanism by which an increase in arterial stiffness may increase paroxysmal AF, one possibility is that enhanced LV load and hypertrophy caused by an increase in the augmentation index may elevate pressure and enlarge the left atrium, thus maintaining AF. This study showed a positive correlation between the CAVI and the radial augmentation index, suggesting that an increase in arterial stiffness affects LV afterload. In line with our previous studies, ${ }^{16} 22$ this study also found that the

Table 3 Association between the CAVI and other parameters

\begin{tabular}{lrr}
\hline & \multicolumn{2}{l}{ All patients } \\
\cline { 2 - 3 } Dependent variable: CAVI & $\mathbf{r}$ & p Value \\
\hline Age & 0.47 & $<0.01$ \\
Male & 0.01 & 0.86 \\
Body mass index & 0.02 & 0.79 \\
Systolic blood pressure & 0.22 & $<0.01$ \\
Diastolic blood pressure & 0.04 & 0.54 \\
Pulse pressure & 0.29 & $<0.01$ \\
Heart rate & -0.08 & 0.31 \\
Serum creatine & 0.12 & 0.10 \\
LDL cholesterol & -0.10 & 0.16 \\
HDL cholesterol & -0.15 & 0.04 \\
Haemoglobin A1c & 0.06 & 0.44 \\
Left atrial diameter & 0.22 & $<0.01$ \\
LV mass index & 0.32 & $<0.01$ \\
LV ejection fraction & 0.09 & 0.26 \\
\hline CAVI, cardio-ankle vascular index; HDL, high-density lipoprotein; LDL, low-density \\
lipoprotein; LV, left ventricular. & & \\
& &
\end{tabular}


Figure 2 Correlation of cardio-ankle vascular index with left ventricular mass index and left atrial diameter.
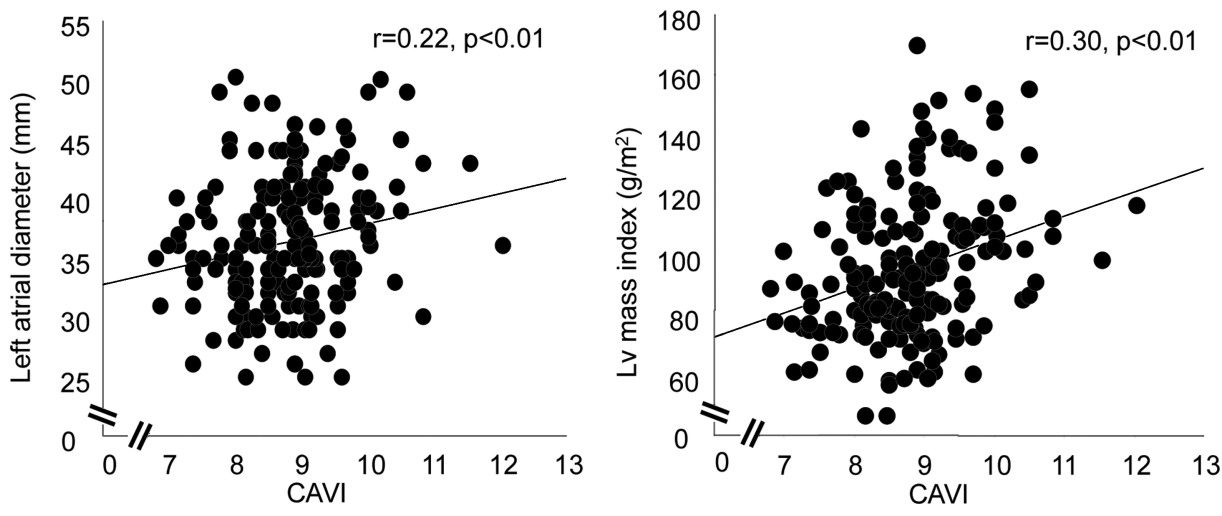

CAVI was positively associated with the LV mass index and left atrial size. Another potential mechanism for the association between arterial stiffness and $\mathrm{AF}$ is that increased arterial stiffness predisposes individuals to a generalised cardiovascular inflammatory response. ${ }^{23}{ }^{24}$ In fact, a case-control study showed that $\mathrm{C}$ reactive protein is elevated in patients with $\mathrm{AF}^{25}$ and high sensitivity $\mathrm{C}$ reactive protein had significant positive association between carotid-femoral PWV even in healthy population. ${ }^{26}$

The CAVI is a widely used non-invasive measure of arterial stiffness with the benefit of being independent of blood pressure. ${ }^{12}$ The CAVI tends to be higher in patients with hypertension than in non-hypertensive patients ${ }^{13}$ and this study also showed a correlation between the CAVI and systolic blood pressure; this, however, does not necessarily mean that the CAVI is dependent on blood pressure. In fact, Shirai et $a l^{27}$ reported that the $\beta 1$-adrenoceptor blocker metoprolol lowers systolic blood pressure but does not decrease the CAVI. Considering the interaction between arterial stiffness and paroxysmal AF, we need to identify the arterial stiffness which is not interfered by blood pressure at the time of measuring. The independence of the CAVI from occasional blood pressure may be advantageous compared with carotid-femoral PWV. Hypertension treatment is one of the most important factors in the prevention of $\mathrm{AF}^{28}$ Therefore, using the CAVI to evaluate the effectiveness of

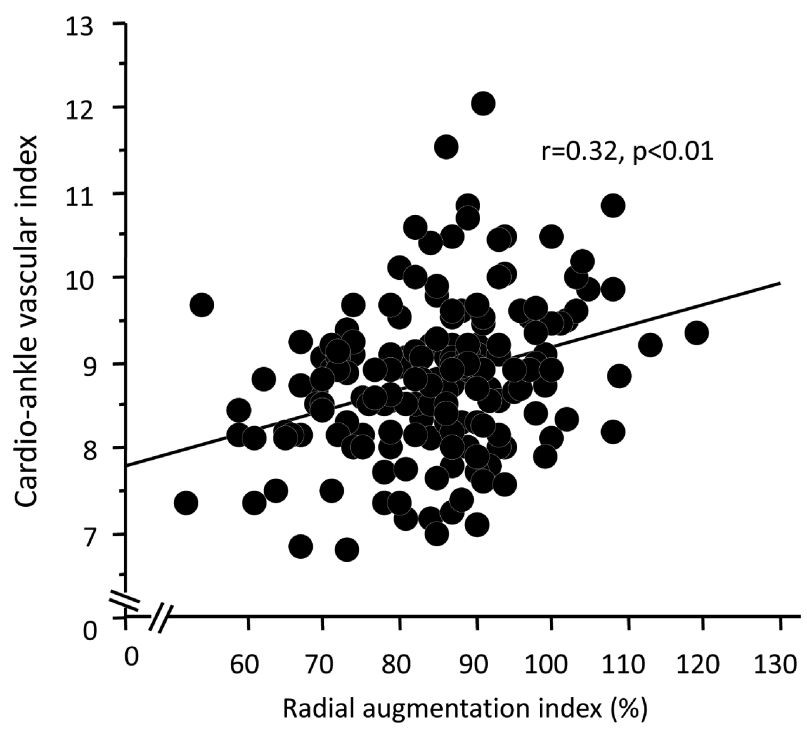

Figure 3 Association between cardio-ankle vascular index and radial augmentation index in all subjects.
Table 4 Association of paroxysmal atrial fibrillation with the CAVI, blood pressure parameters and echocardiographic parameters

\begin{tabular}{llll}
\hline & Adjusted OR & 95\% Cl & p Value \\
\hline Stiffness parameter: CAVI (per 1 unit) & 1.83 & 1.15 to 2.92 & 0.01 \\
Systolic blood pressure (per $20 \mathrm{~mm} \mathrm{Hg}$ ) & 1.35 & 0.89 to 2.04 & 0.16 \\
Diastolic blood pressure (per $10 \mathrm{~mm} \mathrm{Hg}$ ) & 1.21 & 0.84 to 1.74 & 0.3 \\
Pulse pressure (per $10 \mathrm{~mm} \mathrm{Hg}$ ) & 1.23 & 0.94 to 1.62 & 0.13 \\
Left atrial diameter (per $5 \mathrm{~mm}$ ) & 1.84 & 1.25 to 2.72 & $<0.01$ \\
LV mass index (per $20 \mathrm{~g} / \mathrm{m}^{2}$ ) & 1.12 & 0.83 to 1.68 & 0.37 \\
\hline
\end{tabular}

This model included age, gender, heart rate, use of antihypertensive therapy and antiarrhythmic therapy.

CAVI, cardio-ankle vascular index; LV, left ventricular.

hypertensive treatment on vascular properties could further help in prevention of AF. Additional larger-scale prospective studies are warranted to determine the applicability of the CAVI to assessing risk of $\mathrm{AF}$ in the general population.

The present study had several limitations. First, data on smoking, which is known to affect the CAVI, were not obtained from the participants. We cannot rule out the possibility that there were different proportions of smokers in the $\mathrm{AF}$ and control groups. Second, it is known that the use of antihypertensive influences the CAVI. We analysed the relationship between the CAVI and paroxysmal AF in subjects receiving prescription medications; therefore, we cannot reject a possible influence of the medications on paroxysmal AF. Third, because our study was cross-sectional, we could not clearly establish a causal relationship between the CAVI and paroxysmal AF. In addition, participants were enrolled from only two sites. Some selection bias cannot be denied. Prospective multicentre studies are necessary to resolve this issue.

\section{CONCLUSIONS}

In conclusion, this study demonstrated that an increase in the CAVI is independently associated with paroxysmal AF. Our findings indicated that the CAVI might represent a modifiable risk marker for AF.

Contributors TM, MD, YN, YO, KS, SK and YN conceived the study, and participated in its design and coordination and helped to draft the manuscript. $\mathrm{HI}$ was involved in drafting the manuscript or revising it critically.

Competing interests None.

Patient consent Obtained.

Ethics approval The Institutional Review Board of Okayama University.

Provenance and peer review Not commissioned; externally peer reviewed. 


\section{REFERENCES}

1 Benjamin EJ, Wolf PA, D'Agostino RB, et al. Impact of atrial fibrillation on the risk of death: the Framingham Heart Study. Circulation 1998;98:946-52.

2 Go AS, Hylek EM, Phillips KA, et al. Prevalence of diagnosed atrial fibrillation in adults: national implications for rhythm management and stroke prevention: the AnTicoagulation and Risk Factors in Atrial Fibrillation (ATRIA) Study. JAMA 2001;285:2370-5.

3 Benjamin EJ, Levy D, Vaziri SM, et al. Independent risk factors for atrial fibrillation in a population-based cohort. The Framingham Heart Study. JAMA 1994;271:840-4.

4 Psaty BM, Manolio TA, Kuller LH, et al. Incidence of and risk factors for atrial fibrillation in older adults. Circulation 1997;96:2455-61.

5 Wang TJ, Parise $H$, Levy $D$, et al. Obesity and the risk of new-onset atrial fibrillation. JAMA 2004;292:2471-7.

6 Mitchell GF, Vasan RS, Keyes MJ, et al. Pulse pressure and risk of new-onset atrial fibrillation. JAMA 2007;297:709-15.

7 Heeringa J, van der Kuip DA, Hofman A, et al. Subclinical atherosclerosis and risk of atrial fibrillation: the rotterdam study. Arch Intern Med 2007;167:382-7.

8 Chen LY, Foo DC, Wong RC, et al. Increased carotid intima-media thickness and arterial stiffness are associated with lone atrial fibrillation. Int J Cardiol 2013;168:3132-4.

9 Lantelme $P$, Laurent $S$, Besnard C, et al. Arterial stiffness is associated with left atrial size in hypertensive patients. Arch Cardiovasc Dis 2008;101:35-40.

10 Lee $\mathrm{SH}$, Choi $\mathrm{S}$, Jung $\mathrm{JH}$, et al. Effects of atrial fibrillation on arterial stiffness in patients with hypertension. Angiology 2008;59:459-63.

11 Reiffel JA. Is arterial stiffness a contributing factor to atrial fibrillation in patients with hypertension? A preliminary investigation. Am J Hypertens 2004;17:213-16.

12 Shirai K, Utino J, Otsuka K, et al. A novel blood pressure-independent arterial wall stiffness parameter; cardio-ankle vascular index (CAVI). J Atheroscler Thromb 2006;13:101-7.

13 Shirai $\mathrm{K}$, Hiruta N, Song M, et al. Cardio-ankle vascular index (CAVI) as a novel indicator of arterial stiffness: theory, evidence and perspectives. J Atheroscler Thromb 2011;18:924-38.

14 Murgo JP, Westerhof N, Giolma JP, et al. Aortic input impedance in normal man: relationship to pressure wave forms. Circulation 1980;62:105-16.

15 Mitchell GF, Lacourciere Y, Arnold JM, et al. Changes in aortic stiffness and augmentation index after acute converting enzyme or vasopeptidase inhibition. Hypertension 2005;46:1111-17.

16 Kaji Y, Miyoshi T, Doi M, et al. Augmentation index is associated with B-type natriuretic peptide in patients with paroxysmal atrial fibrillation. Hypertens Res 2009;32:611-16
17 Doi M, Miyoshi T, Hirohata S, et al. Increased augmentation index of the radial pressure waveform in patients with paroxysmal atrial fibrillation. Cardiology 2009;113:138-45.

18 Fuster V, Ryden LE, Cannom DS, et al. ACC/AHA/ESC 2006 guidelines for the management of patients with atrial fibrillation--executive summary: a report of the American College of Cardiology/American Heart Association Task Force on Practice Guidelines and the European Society of Cardiology Committee for Practice Guidelines (Writing Committee to Revise the 2001 Guidelines for the Management of Patients With Atrial Fibrillation). J Am Coll Cardiol 2006;48:854-906.

19 Miyoshi T, Doi M, Hirohata S, et al. Cardio-ankle vascular index is independently associated with the severity of coronary atherosclerosis and left ventricular function in patients with ischemic heart disease. J Atheroscler Thromb 2010;17:249-58.

20 Cheitlin MD, Armstrong WF, Aurigemma GP, et al. ACC/AHA/ASE 2003 Guideline Update for the Clinical Application of Echocardiography: summary article. A report of the American College of Cardiology/American Heart Association Task Force on Practice Guidelines (ACC/AHA/ASE Committee to Update the 1997 Guidelines for the Clinical Application of Echocardiography). J Am Soc Echocardiogr 2003;16:1091-110.

21 Devereux RB, Reichek N. Echocardiographic determination of left ventricular mass in man. Anatomic validation of the method. Circulation 1977:55:613-8.

22 Sakane K, Miyoshi T, Doi M, et al. Association of new arterial stiffness parameter, the cardio-ankle vascular index, with left ventricular diastolic function. J Atheroscler Thromb 2008;15:261-8.

23 Chae CU, Lee RT, Rifai N, et al. Blood pressure and inflammation in apparently healthy men. Hypertension 2001;38:399-403.

24 Tsioufis C, Dimitriadis K, Selima M, et al. Low-grade inflammation and hypoadiponectinaemia have an additive detrimental effect on aortic stiffness in essential hypertensive patients. Eur Heart J 2007;28:1162-9.

25 Chung MK, Martin DO, Sprecher D, et al. C-reactive protein elevation in patients with atrial arrhythmias: inflammatory mechanisms and persistence of atrial fibrillation. Circulation 2001;104:2886-91.

26 Wang X, Du Y, Fan L, et al. Relationships between HDL-C, hs-CRP, with Central Arterial Stiffness in Apparently Healthy People Undergoing a General Health Examination. PLOS ONE 2013;8:e81778.

27 Shirai $K$, Song M, Suzuki J, et al. Contradictory effects of beta1- and alpha1aderenergic receptor blockers on cardio-ankle vascular stiffness index (CAVI)--CAVI independent of blood pressure. J Atheroscler Thromb 2011;18:49-55.

28 Haywood LJ, Ford CE, Crow RS, et al. Atrial fibrillation at baseline and during follow-up in ALLHAT (Antihypertensive and Lipid-Lowering Treatment to Prevent Heart Attack Trial). J Am Coll Cardiol 2009;54:2023-31. 